\title{
La Teoría de la Organización Requerida de Elliott Jaques: el capítulo faltante en los libros de texto de administración*
}

\section{Elliott Jaques' Required Organization Theory: The missing chapter in management textbooks}

\section{A Teoria da Orgaanização Requerida de Elliott Jaques: O Capítulo Faltante nos Livros de Texto de Administração}

Recibido: 5 de abril de 2017 Revisado: 9 de mayo de 2017 Aceptado: 11 de mayo de 2017

Dr. Javier Muñoz Orozco***

Universidad Vasco de Quiroga

\section{RESUMEN}

La administración como ciencia es relativamente reciente en el repertorio del conocimiento humano. Algunos autores la ubican a partir del discurso de Henry Robinson Towne en 1886, titulado El ingeniero como economista, dentro de la corriente de la administración científica (George y Álvarez, 2005). A partir de entonces, los libros de texto de administración dan fe de la obra de numerosos autores y escuelas del pensamiento administrativo, pero han pasado por alto la obra de Elliott Jaques (1917-2003), quien desarrolló la Teoría de la Organización Requerida. En este artículo se presenta un resumen de algunos aspectos importantes de dicha teoría, y se hace una comparación siguiendo el método de Glaser y Strauss (1967) de comparaciones constantes, con modelos previos construidos

* $\quad$ Artículo de reflexión. DOI: http://dx.doi.org/10.15332/s2145-1389.2017.0001.07

* Doctor en Ciencias Administrativas. Magíster en Administración. Licenciado en Economía. Coordinador de posgrados Universidad Vasco de Quiroga. Morelia, México. Correo electrónico: jamunoro@hotmail.com. 
sobre bases científicas que impactaron el mundo de la administración, concluyendo que la Organización Requerida debe ser difundida en las escuelas de administración y en las empresas, pues es un modelo de actualidad que engloba a toda la organización y que puede ser proyectado hacia el futuro.

Palabras clave: organización requerida, Teoría de los Sistemas Estratificados.

\section{ABSTRACT}

Management as a science is a relatively newcomer in the human repository. Some authors give the date of its birth in 1886 with Henry Robinson Towne's lecture The engineer as an economist within the context of the Scientific Administration (George \& Alvarez, 2005). Management theories developed and evolved since then and had been published in textbooks for the use of students. However, none of these textbooks offers a glimpse of Elliott Jaques' (19172003) Requisite Organization. This article presents a Requisite Organization's overview and makes a comparative analysis with other major scientific management theories developed in the past, following Glaser and Strauss (1967) method of continuous comparisons. The conclusion is that Jaques' research of more than 60 years should be known in the academic world and taught in business schools.

Keywords: Requisite organization, Stratified Systems Theory.

\section{RESUMO}

A administração como ciência é relativamente nova no repertório do conhecimento humano. Alguns autores a colocam a partir do discurso de Henry Robinson Towne em 1886, intitulado 0 Engenheiro como Economista, dentro da atual corrente da Administração Científica (George e Álvarez, 2005). A partir de então, os livros de administração que dão crédito às obras de inumeráveis autores e escolas do pensamento administrativo, mas não consideram o trabalho de Elliott Jaques (1917-2003) que desenvolveu a teoria da organização requerida. Este artigo resume alguns aspectos importantes desta teoria e é comparada mediante o modelo de Glaser e Strauss (1967/2006) de comparações constantes, com modelos prévios construídos em bases científicas que impactaram o mundo da administração, concluindo que a organização requerida deve ser difundida nas escolas de administração e nas empresas dado que é um modelo atual que inclui toda a organização e pode ser projetada para o futuro.

Palavras-chave: organização requerida, alcance temporal, capacidade de processamento mental, teoria dos sistemas estratificados.

\section{INTRODUCCIÓN}

La Teoría de la Organización Requerida del doctor canadiense Elliott Jaques es poco conocida en América Latina, con excepción de Argentina, a raíz de los contactos sostenidos por el doctor Jaques con algunos de sus discípulos de ese país en la Universidad de Brunel en Inglaterra, y las visitas del propio Jaques a Argentina. Pero es igualmente poco conocida en Canadá, en los Estados Unidos, en Australia y en Europa, lugares en donde el autor ejerció sus más de sesenta años de vida profesional dedicada a la investigación y a la consultoría.

Jaques fue galardonado por el general Colin Powell, en nombre de las fuerzas armadas norteamericanas, por su extraordinaria contribución en el campo de la 
teoría del liderazgo militar y por la capacitación otorgada a todos los departamentos de servicio público de los Estados Unidos (ROII, 2017). En el año 2000 recibió el premio Harry Levinson de la American Psychological Foundation, por una vida entera de contribuciones científicas.

Como miembro fundador del Instituto Tavistock de Relaciones Humanas, llevó a cabo una intervención en la empresa Glacier Metal en el año de 1947, misma que se prolongó por más de tres décadas y dio nacimiento a la Teoría de la Organización Requerida, que ha impactado los ámbitos de la psicología y de las ciencias administrativas. En 1964 fue invitado a desarrollar la primera escuela de ciencias sociales interdisciplinaria en la Universidad de Brunel, y en 1970 estableció el Instituto para la Investigación de la Organización y las Ciencias Sociales de la misma universidad (BIOSS).

Fue autor de más de 20 libros, entre los que podemos mencionar Requisite organiæation (1989, 1996, 1998, 2006), Human capability (1994), Social power and the CEO (2002), Life and behavior of living organisms (2002), A general theory of bureaucracy (1976) y Creativity and work (1970, 1990).

¿Por qué entonces una obra tan importante y monumental no es objeto de estudio en las escuelas de administración?

Quizá dos razones pueden dar una pista para esta incógnita. La primera es que Jaques fue un abogado de la teoría burocrática (Jaques, 2000) dentro de la línea de Max Weber y la corriente burocrática, y si algo es común a la mayoría de las personas de este planeta es su aborrecimiento por los sistemas burocráticos, y su escaso entendimiento de los postulados que los sostienen y de la aplicación correcta de esta teoría.

En segundo lugar, la Teoría de la Organización Requerida está fundamentada sobre bases científicas, a menudo complicadas de entender, como es el caso de la complejidad del procesamiento mental. No son recetas fáciles de implementar y puede suceder que muchos aspirantes a puestos directivos se vean confrontados por la teoría ante su propia incapacidad de escalar a estos puestos. Sea cual fuere la razón de este desconocimiento, la obra de Jaques es imprescindible en la biblioteca del administrador. Por ende, el propósito de este artículo es difundir la extraordinaria labor de Jaques para que ocupe un lugar en el acervo del conocimiento administrativo y sea incluida en los textos que forman a los estudiantes de las ciencias administrativas.

\section{LA TEORÍA DE LA ORGANIZACIÓN REQUERIDA}

Jaques empieza por definir los componentes de la organización, contando en primer lugar con la estructura social, que comprende su jerarquía ejecutiva, su sistema consultivo y su sistema de promoción, así como el conjunto de roles que son llevados a cabo por los individuos y los grupos. En segundo lugar, aparece la cultura empresarial, que se define como la forma acostumbrada de pensar y hacer las cosas, que es compartida por la mayoría de los individuos y que los nuevos reclutas deben endosar para formar parte de la organización (Jaques, 1951). Cabe señalar que Jaques acuñó el término Cultura Organizacional en su libro de 1951 The changing culture of a factory, obra con la cual obtuvo su doctorado en Harvard, y es el registro más antiguo que hemos encontrado del término.

El tercer componente de la organización es la personalidad de los miembros, es el Yo del individuo, que incluye sus actitudes y creencias, sus deseos y sus decisiones. Para hacer cambios en las organizaciones, 
es necesario intervenir en estos tres pilares, tomar en consideración las modificaciones que se deban hacer en la estructura, en la cultura y en los ajustes de orden personal en los interesados.

Para Jaques, las organizaciones son un reflejo de la naturaleza, por lo que una adecuada estructura, acorde a la naturaleza del ser humano y de la organización, produce un comportamiento ideal de los empleados en el trabajo, de ahí que la intervención psicológica no sea necesaria cuando se tiene una organización requerida por la naturaleza. El autor, como miembro fundador del Instituto Tavistock, fue invitado por Wilfred Brown, entonces director de la fábrica Glacier Metal, para llevar a cabo una investigación que consistía en un estudio de tipo psicosocial en el marco de la corriente psicoanalítica predominante en Tavistock. Sin embargo, al pasar de los años, y después de la publicación del primer reporte del estudio, el autor reconoció que no era necesaria la intervención psicológica en una empresa cuyos tres pilares se muestran sólidamente constituidos, y se desligó del movimiento del desarrollo organizacional para concentrarse en la formulación y desarrollo de lo que más adelante sería la Teoría de la Organización Requerida.

La piedra fundamental de esta teoría es la Jerarquía de Responsabilidad Gerencial (MAH), por sus siglas en Inglés (Managerial Accountability Hierarchy, que también se traduce como Jerarquía Directiva de Rendición de Cuentas), ya que asevera el autor que la organización es un sistema que emplea personal para que despliegue sus talentos en el trabajo que realiza y es remunerado por su efectividad personal dentro de las circunstancias prevalecientes que le hacen producir resultados (Jaques, 2006). La dirección juega un papel preponderante, ya que al directivo se le solicitan cuentas por los resultados de otros, por sostener un equipo capaz de producir tales resultados y por realizar un liderazgo efectivo.
Es importante señalar la diferencia que existe entre la MAH y la Asociación, ya que la Organización Requerida solamente se aplica a la MAH, entendiendo a esta como la parte operativa y jerárquica de cualquier organización, mientras que la Asociación está compuesta por miembros del mismo nivel y tiene sus propios sistemas de gobernanza. En la Asociación los directivos son elegidos, mientras que en la MAH los ocupantes de los diferentes puestos son asignados (Jaques, 2000). La cámara de diputados, de senadores, los cuerpos colegiados de las universidades, los sínodos de obispos, los consejos de administración y asamblea de socios de cualquier organización, son ejemplos de Asociación; mientras que toda la estructura operativa de una organización para la producción de bienes y servicios, dese el CEO hasta el operario de línea, e igualmente los funcionarios y empleados de las oficinas de gobierno, son ejemplos de MAH.

\section{LOS COMPONENTES DE LA ORGANIZACIÓN REQUERIDA}

Son cuatro los componentes de la organización requerida, cada uno de los cuales se despliega profusamente hasta el detalle y es objeto de estudios científicos que corroboran su existencia. En primer lugar, el descubrimiento más importante que llevó a Jaques a entender el correcto funcionamiento de la Jerarquía Directiva de Rendición de Cuentas (MAH), fue el de colocar a la persona correcta en el puesto correcto. Segundo, una estructura organizacional universalmente aplicable, con un sistema jerárquico de estratos administrativos, un sistema de rendición de cuentas, un sistema de autoridad en relaciones laterales de trabajo, equipos de trabajo con líderes responsables y funciones específicas con cada nivel de la organización. Tercero, un sistema detallado de procesos de mando. Cuarto, una 
estructura equitativamente diferenciada con niveles de sueldo equitativos ligados a la estructura de los estratos jerárquicos.

\section{PONER A LA PERSONA CORRECTA EN EL LUGAR CORRECTO}

Tiempo después de haber iniciado la intervención en Glacier Metal, Jaques recibió en una ocasión a un pequeño grupo de obreros que querían consultar con él acerca de algo que no entendían. Para ellos era una incógnita el hecho de que a diario llegaban a trabajar con su overol y lentes para producir un determinado monto de balines para rodamientos con determinadas características de calidad, y al final de la semana se les remuneraba con base en una tarifa diaria. El supervisor, por su parte, tenía una oficina con aire acondicionado en el fondo del taller, llegaba a trabajar con un overol de calidad y lentes de marca, no sabían qué hacía en esa oficina y de vez en cuando se acercaba a la línea de producción para supervisar su funcionamiento. Por este trabajo el supervisor recibía una remuneración mucho mayor y con base en una tarifa anual aplicada quincenalmente. ¿Por qué? Esta consulta fue para Jaques como una epifanía, pues le dio la pauta de que los talentos de las personas son variados, y ese talento se puede medir, como el sueldo, por día, por mes o por años. Después de cavilar sobre el tema, el autor llegó al descubrimiento de lo que llamó el Alcance Temporal de la Discreción (Time-Span of Discretion, también traducido como Horizonte Temporal. En francés ha sido traducido como Période d'Autonomie, término que describe el mayor de los atributos del alcance temporal), que es el tiempo máximo que una persona visualiza para completar su tarea más larga, sin dirección y utilizando su propio criterio. Realizar un trabajo en estos términos requiere de aplicar un esfuerzo psicológico, de soportar la incertidumbre y hacer frente a la responsabilidad, en un grado acorde a la magnitud del tiempo empleado. Para comprender a cabalidad la profundidad del concepto alcance temporal, conviene tomar en cuenta los siguientes atributos:

1. Entre más largo es el tiempo de que una persona dispone de autonomía sin que sus resultados sean evaluados, más grande es el esfuerzo psicológico requerido por la tarea.

2. A mayor tiempo de soportar la incertidumbre y aun así continuar con el trabajo, mayor es la responsabilidad.

3. A mayor tiempo que la organización deja ejercer la autonomía de un individuo, tomar decisiones y destinar recursos, más grande es la confianza que se le tiene y mayor es la responsabilidad que se le confiere.

Con base al alcance temporal se establece el nivel de trabajo de la persona, y se presenta como una serie de estratos, tal como figura en la tabla 1.

Tabla 1. Niveles de trabajo

\begin{tabular}{|l|l|c|}
\hline $\begin{array}{c}\text { Alcance } \\
\text { temporal }\end{array}$ & \multicolumn{1}{|c|}{ Niveles de trabajo } & Estrato \\
\hline $\begin{array}{l}10 \text { años } \\
7 \text { años } \\
5 \text { años }\end{array}$ & Presidente de unidad de negocios & SV \\
\hline $\begin{array}{l}4 \text { años } \\
3 \text { años } \\
2 \text { años }\end{array}$ & Director general, COO, CFO, CIO & SIV \\
\hline $\begin{array}{l}20 \text { meses } \\
16 \text { meses } \\
12 \text { meses }\end{array}$ & $\begin{array}{l}\text { Director de unidad, director de planta, } \\
\text { director de operaciones }\end{array}$ & SIII \\
\hline $\begin{array}{l}9 \text { meses } \\
6 \text { meses } \\
3 \text { meses }\end{array}$ & $\begin{array}{l}\text { Supervisor, coordinador, administrador } \\
\text { de proyectos, ingeniero, jefe de línea }\end{array}$ & SII \\
\hline $\begin{array}{l}1 \text { mes } \\
1 \text { semana } \\
1 \text { día }\end{array}$ & $\begin{array}{l}\text { Técnico, operador de equipo, } \\
\text { empleado de oficina, capturista }\end{array}$ & SI \\
\hline
\end{tabular}

Fuente: Foster (2016) 
Aunado al alcance temporal, Ja ques descubrió años después la capacidad de procesamiento mental. Siguiendo la línea marcada por Piaget en el desarrollo mental de los niños, Jaques y Cason elaboraron un esquema de desarrollo de la capacidad de procesamiento mental en adultos que contempla, por un lado, el método de procesamiento, que puede ser declarativo, acumulativo, serial o paralelo; por otro lado, la complejidad del orden de procesamiento mental que puede ser simbólico, abstracto-conceptual, o universal. En cada uno de los tres órdenes de complejidad se insertan los cuatro métodos de procesamiento mental, como se muestra en la tabla 2, en donde se combinan ambas variables. Aparece también un primer orden de complejidad que es el concreto, propio de los niños y adolescentes, sin embargo, asevera Jaques que para ejecutar las labores diarias de las organizaciones es necesario haber madurado en la edad adulta hacia el orden simbólico (Jaques y Cason, 2013) que es el mundo de los términos y del significado de las palabras, o hacia el orden abstractoconceptual que es el orden de los modelos complejos.

El orden universal es propio de mentes muy complejas y privilegiadas que pueden elaborar nuevas teorías que marcan las pautas seguidas por la humanidad, tales como Armand Hammer o Matsushita Konosuke. Por lo tanto, las actividades diarias de las organizaciones se llevan a cabo por personas cuya capacidad se encuentra en el orden simbólico o en el orden abstracto-conceptual.

Tabla 2. Categorías de complejidad del procesamiento mental

\begin{tabular}{|l|l|l|}
\hline Categoría & $\begin{array}{c}\text { Complejidad del orden de la } \\
\text { información }\end{array}$ & $\begin{array}{c}\text { Método de } \\
\text { proceso } \\
\text { mental }\end{array}$ \\
\hline D4 & Cuarto orden universal & Paralelo \\
D3 & “ & Serial \\
D2 & " & Acumulativo \\
D1 & Declarativo \\
\hline
\end{tabular}

\begin{tabular}{|l|l|l|}
\hline Categoría & $\begin{array}{c}\text { Complejidad del orden de la } \\
\text { información }\end{array}$ & $\begin{array}{c}\text { Método de } \\
\text { proceso } \\
\text { mental }\end{array}$ \\
\hline C4 & Tercer orden abstracto- & Paralelo \\
C3 & conceptual & Acrial \\
C2 & " & Declarativo \\
C1 & " & Paralelo \\
\hline B4 & Segundo orden simbólico & Serial \\
B3 & " & Acumulativo \\
B2 & " & Declarativo \\
B1 & Parale \\
\hline A4 & Primer orden concreto & Paralo \\
A3 & " & Serial \\
A2 & " & Acumulativo \\
A1 & Declarativo \\
\hline
\end{tabular}

Fuente: Jaques y Cason (2013)

Cada persona posee de nacimiento una determinada ruta de maduración de su capacidad de procesamiento mental a partir del orden concreto declarativo. Después de la adolescencia y alrededor de los 20 años de edad, la maduración se da en el orden simbólico, hay quienes sobrepasan el orden simbólico y llegan al abstracto-conceptual, algunos genios pueden llegar al orden universal.

Según datos estadísticos reportados por Tom Foster, el $80 \%$ de la población laboral se mueve en su maduración a través de los años dentro de bandas ascendentes desarrolladas por Jaques y Cason que se desplazan sobre los estratos I y II exclusivamente (B1 declarativo simbólico y B2 acumulativo simbólico de la tabla 2); considerando que un $10 \%$ de la población no es empleable, solamente queda un $10 \%$ que se desplaza entre los estratos III a VIII durante su vida laboral entre los 20 y 70 años de edad (Foster, 2016).

En un estudio realizado por Jaques y Cason en empresas exitosas de Australia y Estados Unidos (Jaques y Cason, 2013), en donde se observaba un correcto funcionamiento de los directivos en sus respectivos 
puestos, se encontró una correlación de 0.98 entre el alcance temporal y la capacidad de procesamiento mental de una persona, lo cual establece las bases científicas de esta teoría. El significado de este hallazgo implica que si una persona tiene un horizonte temporal de dos a cuatro años, existe un $98 \%$ de probabilidad de que su método de procesamiento mental sea el paralelo, dentro de la complejidad de información correspondiente al orden simbólico (B4). Jaques considera que cualquier jefe debe estar un estrato por encima de sus subordinados para ser una auténtica guía a la que acuden en busca de orientación. Ahora bien, hay que recordar que tanto el alcance temporal como la capacidad de procesamiento mental son dinámicos dentro de la persona, pues se encuentran en constante movimiento dentro de una banda ascendente a lo largo de los años y que es diferente para cada individuo. De ahí la necesidad de contar en los departamentos de Recursos Humanos con un seguimiento constante de la maduración del pool de talentos para asegurar que la jerarquía conserve los principios de adecuación del personal.

En estudios posteriores se investigó el nivel de remuneraciones que las personas consideraban adecuadas en el desempeño de sus actividades y se encontró que existe una progresión geométrica en los sueldo de los diferentes estratos (Jaques, 2006).

Todas las variables que se han explicado hasta aquí aparecen en la tabla 3 que contiene la congruencia entre los estratos, llamada Teoría de los Sistemas Estratificados y sintetiza el pensamiento medular de la teoría.

Para Jaques, la Capacidad Potencial es la máxima capacidad de procesamiento mental a la que puede llegar un individuo en su proceso de maduración, mientras que la Capacidad Aplicada es la que despliega en el momento actual. Según Tom Foster (2016), la capacitación y los niveles educativos universitarios inciden en colocar la Capacidad Aplicada en el extremo alto de la banda de maduración.

Tabla 3. Teoría de los Sistemas Estratificados. Congruencia entre los estratos

\begin{tabular}{|c|c|c|c|c|}
\hline Estrato & $\begin{array}{l}\text { Alcance } \\
\text { temporal }\end{array}$ & $\begin{array}{c}\text { Capacidad de } \\
\text { procesamiento } \\
\text { mental }\end{array}$ & $\begin{array}{c}\text { Nivel } \\
\text { jerárquico }\end{array}$ & $\begin{array}{c}\text { Paga } \\
\text { equitativa }\end{array}$ \\
\hline VIII & 50 años & $\begin{array}{l}\text { C4 Abstracto- } \\
\text { Conceptual } \\
\text { Paralela } \\
\end{array}$ & $\begin{array}{l}\text { CEO } \\
\text { Multinacional }\end{array}$ & $32 X$ \\
\hline VII & 20 años & $\begin{array}{l}\text { C3 Abstracto- } \\
\text { Conceptual } \\
\text { Serial }\end{array}$ & $\begin{array}{l}\text { CEO } \\
\text { Corporativo }\end{array}$ & $16 \mathrm{X}$ \\
\hline VI & 10 años & $\begin{array}{l}\text { C2 Abstracto- } \\
\text { Conceptual } \\
\text { Acumulativa }\end{array}$ & $\begin{array}{l}\text { Vicepresidente } \\
\text { Ejecutivo }\end{array}$ & $8 \mathrm{X}$ \\
\hline V & 5 años & $\begin{array}{l}\text { C1 Abstracto- } \\
\text { Conceptual } \\
\text { Declarativa }\end{array}$ & $\begin{array}{l}\text { Director de } \\
\text { Unidad de } \\
\text { Negocios }\end{array}$ & $4 X$ \\
\hline IV & 2 años & $\begin{array}{l}\text { B4 Simbólica } \\
\text { Paralela }\end{array}$ & $\begin{array}{l}\text { Gerente de } \\
\text { Planta }\end{array}$ & $2 X$ \\
\hline III & 1 año & $\begin{array}{l}\text { B3 Simbólica } \\
\text { Serial }\end{array}$ & $\begin{array}{l}\text { Gerente de } \\
\text { Área }\end{array}$ & $\mathrm{X}$ \\
\hline II & 3 meses & $\begin{array}{l}\text { B2 Simbólica } \\
\text { Acumulativa }\end{array}$ & $\begin{array}{l}\text { Gerente de } \\
\text { línea }\end{array}$ & $.55 \mathrm{X}$ \\
\hline I & 1 día & $\begin{array}{l}\text { B1 Simbólica } \\
\text { Declarativa }\end{array}$ & Operario & $.31 \mathrm{X}$ \\
\hline
\end{tabular}

Fuente: Jaques (2006)

Pero la capacidad de procesamiento mental aunada al alcance temporal no lo es todo, se requiere, para desempeñarse adecuadamente en la línea jerárquica de la organización requerida, que se cumpla la ecuación de la Capacidad Aplicada según la fórmula $\mathrm{CA}=$ $\mathrm{CPM}+\mathrm{V}+\mathrm{KS}-\mathrm{T}$.

En esta ecuación la Capacidad Aplicada CA está en función de la capacidad de procesamiento mental CPM; más la valoración del individuo por su trabajo $\mathrm{V}$ (algo similar a adoptar el factor motivacional de Herzberg (1966) llamado Trabajo en Sî); más el 
dominio de los aspectos cognitivos, de las habilidades y las competencias que demanda su puesto KS; y además, que desde el punto de vista de su comportamiento en las relaciones interpersonales no tenga taras de personalidad $\mathrm{T}$ que le resten méritos a su gestión. Es entonces que el directivo se puede llamar un verdadero jefe, aquel a quien acuden los subordinados en busca de orientación, quien les asigna sus tareas y evalúa su desempeño, estableciendo un contexto adecuado de trabajo, ni muy estrecho, ni muy laxo. En este contexto, Jaques asegura que no es necesario que su jefe le caiga bien, o que su subordinado no sea su amigo, el trabajo será fluido y los problemas interpersonales estarán ausentes.

\section{OTROS COMPONENTES DE LA ORGANIZACIÓN REQUERIDA}

Los tres componentes restantes (estructura, sistemas de mando y paga equitativa) se apegan en gran medida a los dictados de la teoría burocrática y son de dominio general, sin embargo, Jaques aplica en cada uno de ellos su estratificación de los niveles de trabajo, lo cual los convierte en sistemas lógicos y apegados a la razón.

Cabe señalar, dentro del sistema detallado de procesos de mando, el liderazgo de tres niveles vía acompañamiento, desarrollo de carrera, análisis y desarrollo de talento (Sistema mor sor que significa Manager once removed y Subordinate once removed. En el primer caso se trata del jefe del jefe, en el segundo, del subordinado del subordinado). Este liderazgo abarca a tres estratos de la jerarquía, el estrato A es el más alto, le sigue el B y luego el C. Consiste en que si A le pide a $B$ cuentas por los resultados de $\mathrm{C}$, entonces $\mathrm{B}$ debe tener la mínima autoridad requerida con respecto a $\mathrm{C}$, en primer lugar para vetar a $\mathrm{C}$ por juzgar que no tiene las competencias del puesto, para decidir el tipo de trabajo que le será asignado, para decidir la evaluación de eficiencia y para decidir el inicio de remoción en caso de incompetencia o desempeño deficiente. $\mathrm{C}$ y A pueden tener un canal directo de comunicación siempre y cuando B esté al tanto. No es necesario que forzosamente $\mathrm{C}$ tenga que pasar por $\mathrm{B}$ para acceder a $\mathrm{A}$. La razón es que $\mathrm{C}$ se encuentra dentro del pool de talento y es un candidato para reemplazar a $B$, una vez que $B$ debido a la maduración de su capacidad esté listo para ser promovido (Jaques, 2006).

También encontramos en los procesos de mando que existen funciones de interrelación entre estratos y entre funciones que hacen que la organización burocrática tenga agilidad y eficiencia en sus procesos. Esto es de suma importancia, ya que muchos de los problemas de estas organizaciones derivan del énfasis exagerado en las atribuciones de poder y las funciones de los departamentos y en la línea jerárquica, que tienden a convertirlos en feudos inexpugnables que dificultan el desempeño general. Los componentes del rol transfuncional de iniciativa de tarea pretenden dar las pautas para la interrelación entre departamentos y entre jerarquías. Se despliegan de la siguiente forma:

El rol colateral, se aplica a los subordinados de un mismo jefe y es la esencia del trabajo en equipo de tipo colaborativo en ausencia del jefe, se espera de los colegas que tomen acciones para mejorar la productividad basándose en las indicaciones recibidas.

El rol de asesoría, se asigna a expertos específicos con autoridad y responsabilidad para tomar iniciativa de ofrecer su asesoría y consejos bajo la forma de consejo no solicitado. Si este ofrecimiento no es aceptado, el asesor busca a alguien más a quien asesorar. 
El rol de recibir o dar servicio, se enmarca dentro de la necesidad que tienen los empleados de recibir servicios para el cumplimiento de la tarea, por parte de proveedores internos o contratistas externos. Como ejemplos tenemos los servicios de contabilidad y auditoría, materiales de oficina, materias primas, instrumentos, uniformes, etc. Se espera que cada puesto tenga muy claro los tipos de servicio que requiere o que puede dar a otros departamentos y otros niveles jerárquicos.

El rol de monitor es el segundo nivel de autoridad de un experto para aconsejar a otra persona, para persuadirla de modificar, mejorar sus prácticas y recomendar nuevas políticas o prácticas cuando sea necesario. Para esto, el monitor debe estar informado acerca de las actividades de los monitoreados y llevar un seguimiento de su desempeño.

En cuanto a la coordinación transfuncional, su papel es hacer trabajar conjuntamente a un cierto número de personas que no son subordinadas del mismo jefe, ya sea en un proyecto específico o como parte de un proceso que requiere participación interdepartamental. Un coordinador dirige a un equipo de trabajo, que puede ser una fuerza de tarea, cuyos miembros provienen de diferentes departamentos.

El rol de auditar, se ejerce por parte de una persona que tiene la autoridad y la responsabilidad de detener el trabajo de otra, con el fin de mantener la calidad de los procesos y de los productos o servicios. El auditor no tiene autoridad para instruir a otro acerca de su trabajo, pero sí la tiene para ingresar a su trabajo e inspeccionar que se lleve a cabo de acuerdo a los procesos establecidos.

Finalmente, el rol de prescribir, es el nivel más alto de autoridad en las relaciones interdepartamentales, en donde una persona posee la autoridad y la responsabilidad de requerir a otra que haga algo y esa persona tiene que obedecer. Por lo general la prescripción se da en los casos de procesos peligrosos que requieren de límites precisos en donde los expertos realizan revisiones y hacen prescripciones con toda autoridad.

\section{LA ADMINISTRACIÓN COMO UNA CIENCIA}

Hasta aquí se ha realizado una somera presentación de algunos puntos básicos de la obra de Jaques, solamente para subrayar la importancia de sus aportaciones al terreno de la administración. A continuación, siguiendo los lineamientos de Glaser y Strauss (1967), relativos al método comparativo, se realizará una comparación de la obra de Jaques con la de dos destacados exponentes de teorías que en su tiempo dieron fundamento y establecieron los lineamientos de la administración como se conoce hoy en día.

Estos autores han sostenido, al igual que Jaques, que sus descubrimientos derivan de la aplicación del método científico, tal es el caso, en primer lugar, de los Principios de la Administración Científica de Frederick Taylor, quien encabezó el movimiento que transformó los talleres de todo el mundo en sitios de trabajo eficiente y productivo (Taylor 1972). Los inventos en tecnología de corte del acero y el modelo de la administración científica han tenido un gran impacto en el desempeño de las organizaciones hasta nuestros días, a través de los ingenieros industriales y la perfección de la ergonomía, que conecta al ser humano con la tecnología.

Taylor (1972) consideraba que el remedio contra la ineficiencia era la administración sistemática y que "la mejor administración es una verdadera ciencia, basada en principios, reglas y leyes claramente definidos, que le sirven de cimientos" (p. 17). 
En segundo lugar, Fayol (1977) con su Administración Industrial y General, también habla del método científico en su obra como una "doctrina consagrada, conjunto de principios, reglas métodos y procedimientos aplicados y probados por la experiencia pública” (p. 151), pero critica a Taylor en la forma de llevar a cabo el ejercicio del mando sobre los empleados, poniendo en duda el sistema de supervisión funcional descrito por este. Fayol además es el primero en escribir acerca de los elementos de la administración llamados posteriormente el Proceso Administrativo, y propone 14 principios para llevar a cabo el ejercicio de la administración, por lo que su obra ha sido comparada en profundidad a la de Taylor y puestas una junto a la otra; constituyen para los libros de texto lo que han denominado la Administración Clásica.
Por su parte, Jaques (2006) asevera que "es necesario un fundamento científico sólido sobre el cual construir una organización efectiva de la misma forma en que confiamos con firmeza en las ciencias naturales para nuestros avances tecnológicos" (p. 11), pero se lamenta que en el campo del desarrollo organizacional y de la administración nos encontramos en el mismo estadio de desarrollo que tenían las ciencias naturales a mediados del siglo XVI. Así como la alquimia se convirtió en química cuando se empezó a usar el termómetro, de la misma forma la administración se transforma en una ciencia cuando se usa la herramienta científica llamada Alcance Temporal de la Discreción (Jaques, 2006).

En la tabla 4 se presenta un cuadro comparativo de tres teorías que han impactado al pensamiento administrativo y sus principales características.

Tabla 4. Comparación de teorías administrativas

\begin{tabular}{|c|c|c|c|}
\hline & FREDERICK TAYLOR & HENRI FAYOL & ELLIOTT JAQUES \\
\hline $\begin{array}{l}\text { La administración } \\
\text { como ciencia }\end{array}$ & $\begin{array}{l}\text { Ciencia de los procesos productivos } \\
\text { basada en principios, reglas y leyes } \\
\text { claramente definidos }\end{array}$ & $\begin{array}{l}\text { Doctrina consagrada, conjunto } \\
\text { de principios, reglas métodos y } \\
\text { procedimientos aplicados y probados } \\
\text { por la experiencia pública }\end{array}$ & $\begin{array}{l}\text { Ciencia de la estructura } \\
\text { organizacional: estratificación en } \\
\text { niveles de trabajo con grados de } \\
\text { complejidad ascendente (Teoría de } \\
\text { los Sistemas Estratificados) Ciencia } \\
\text { de los procesos mentales (Capacidad } \\
\text { de procesamiento mental) }\end{array}$ \\
\hline Propósito & $\begin{array}{l}\text { Máxima prosperidad para el patrón y } \\
\text { para los empleados }\end{array}$ & $\begin{array}{l}\text { Preparar a los futuros jefes y al } \\
\text { personal para ejercer con capacidad } \\
\text { sus funciones }\end{array}$ & $\begin{array}{l}\text { Establecer una organización } \\
\text { requerida por la naturaleza } \\
\text { que provea a los ciudadanos } \\
\text { la oportunidad de utilizar sus } \\
\text { capacidades plenas }\end{array}$ \\
\hline Enfoque & Ingeniería & Ingeniería & $\begin{array}{l}\text { Psicología (psicoanálisis) } \\
\text { Modelo burocrático }\end{array}$ \\
\hline \multicolumn{4}{|l|}{$\begin{array}{l}\text { Características del } \\
\text { modelo }\end{array}$} \\
\hline Herramientas & $\begin{array}{l}\text { Cambios en la tecnología de } \\
\text { producción } \\
\text { Estudios de tiempos y movimientos } \\
\text { La idea de labor, reglas de cálculo, } \\
\text { fichas de instrucción }\end{array}$ & $\begin{array}{l}\text { Elementos de la administración: } \\
\text { previsión, organización, dirección, } \\
\text { coordinación y control } \\
14 \text { principios de la administración }\end{array}$ & $\begin{array}{l}\text { Alcance Temporal de la Discreción } \\
\text { Capacidad de procesamiento mental } \\
\text { Niveles de trabajo por estratificación }\end{array}$ \\
\hline
\end{tabular}




\begin{tabular}{|c|c|c|c|}
\hline & FREDERICK TAYLOR & HENRI FAYOL & ELLIOTT JAQUES \\
\hline Alcance & $\begin{array}{l}\text { Una ciencia aplicada a cada proceso } \\
\text { productivo }\end{array}$ & $\begin{array}{l}\text { Elementos de la administración } \\
\text { comunes a todas las organizaciones }\end{array}$ & $\begin{array}{l}\text { Una estructura universalmente } \\
\text { aplicada }\end{array}$ \\
\hline Supervisión & Supervisión funcional & Unidad de mando & $\begin{array}{l}\text { Jerarquía de Responsabilidad } \\
\text { Gerencial (MAH) }\end{array}$ \\
\hline Organización & $\begin{array}{l}\text { El patrón toma el mando y realiza los } \\
\text { estudios científicos } \\
\text { El trabajador recibe capacitación a } \\
\text { través de la supervisión funcional }\end{array}$ & $\begin{array}{l}\text { Proveer a la empresa de materias, } \\
\text { herramientas, capital y personal }\end{array}$ & $\begin{array}{l}\text { Estructura basada en niveles de } \\
\text { trabajo } \\
\text { Procesos de mando bien definidos } \\
\text { Relaciones de rol } \\
\text { Relaciones interfuncionales de } \\
\text { trabajo }\end{array}$ \\
\hline $\begin{array}{l}\text { Incorporación y } \\
\text { permanencia }\end{array}$ & Elección científica del trabajador & $\begin{array}{l}\text { Cada servicio debe tener a la cabeza } \\
\text { un hombre competente y activo, cada } \\
\text { agente debe estar en el lugar en que } \\
\text { puede prestar el mayor número de } \\
\text { servicios }\end{array}$ & $\begin{array}{l}\text { Asignación en el puesto según la } \\
\text { fórmula de la Capacidad Aplicada } \\
\mathrm{CA}=\mathrm{f}(\mathrm{CPM}+\mathrm{V}+\mathrm{KS}-\mathrm{T})\end{array}$ \\
\hline Remuneración & $\begin{array}{l}\text { Remuneración basada en la } \\
\text { productividad }\end{array}$ & $\begin{array}{l}\text { Remuneración equitativa que } \\
\text { fomente el celo recompensando el } \\
\text { esfuerzo útil, sin excesos }\end{array}$ & $\begin{array}{l}\text { Remuneración equitativa por estratos } \\
\text { en progresión geométrica }\end{array}$ \\
\hline
\end{tabular}

Fuente: elaboración propia con información de Taylor (1972); Fayol (1977) y Jaques (2006)

La comparación está desfasada en el tiempo, debido a que Taylor y Fayol escribieron sus obras en la segunda década del siglo XX, mientras que Jaques presentó por primera vez la organización requerida en 1989 con ediciones subsecuentes hasta el 2006 (con tres reimpresiones hasta el 2013, llevadas a cabo por Kathryn Cason), y por lo tanto habla de nuevas realidades que los otros dos autores no experimentaron. Sin embargo, al tratarse de teorías que pretenden ser universales, sí tienen un punto de comparación en cuanto a sus planteamientos y relevancia para la economía de los países, para el desarrollo de las organizaciones, para la psique del individuo y para la convivencia en sociedad.

Frederick Taylor estableció los cimientos de su teoría movido por la necesidad de elevar la productividad de los talleres, y con el propósito de obtener la máxima prosperidad para el patrón y para los empleados (Taylor, 1972). Siguiendo la tendencia propia de la Revolución Industrial de considerar a las utilidades como sinónimo de prosperidad y acorde al espíritu de la ética protestante que inspiró a los primeros capitalistas (Fullerton, 1959). Taylor consideraba de muy buena fe que una mayor remuneración derivada de un incremento en la productividad era la respuesta lógica y racional para satisfacer las necesidades del ser humano y vivir con armonía en sociedad. Anteriormente, Benjamín Franklin, uno de los fundadores de la nación norteamericana, enfatizaba la idea de racionalidad como la obligación de ganar más y más dinero (Fullerton, 1959). Así pues, para la reforma científica del capitalismo comandada por Taylor, todo se desvanecía ante esta necesidad imperiosa del hombre económico de perseguir como único objetivo importante en la vida, la creación de la riqueza para provecho individual, y esta es una tendencia del capitalismo que se ha mantenido y magnificado 
en la época actual del posfordismo. El fordismo se conoce como un modo de producción que provocó una acumulación de capital y elevó la calidad de vida de los trabajadores entre el fin de la segunda guerra mundial e inicios de la década de 1970, inspirado en la política de Henry Ford de duplicar el salario de sus empleados. A partir de 1970 el deterioro de los salarios ha sido tal que gran parte de la población trabajadora se ha depauperado al mismo tiempo que se ha profundizado la brecha de ingresos, dando paso a un modo de producción disfuncional; a esta etapa se le conoce como posfordismo (Vidal, 2013).

La administración científica tuvo tal aceptación por parte de los patrones en todo el mundo, que tras la restauración de la dinastía Meiji y la apertura de Japón a la tecnología occidental, el país del sol naciente la adoptó de inmediato en su proceso de industrialización, y fue tal su arraigo que todavía en la década de 1960 Shigheru Kobayashi (1972) se lamentaba de que "la administración tradicional (administración científica) está creando toda clase de problemas humanos al tratar a los seres humanos como si fueran máquinas o animales domésticos" (p. 7). En Rusia, Lenin se maravilló ante la obra de Taylor, entendió perfectamente los alcances que en cuanto a progreso económico significaba su aplicación, pero llevarla a cabo en la Rusia Soviética, en un país de campesinos y con una ideología opuesta al individualismo occidental, significó una relación de aceptación y rechazo al mismo tiempo; así que cuando se implementó el Taylorismo en la incipiente industria rusa, los resultados no fueron los esperados, pero sí tuvo una fuerte influencia en el curso del socialismo soviético (Peci, 2009).

Fayol pretendía difundir la ciencia de la administración hacia los grupos directivos emanados de la Escuela Politécnica, en donde la enseñanza de las matemáticas se privilegiaba por encima de todo y, por el contrario, se tenía un total desconocimiento de la administración.
La paradoja a la que se enfrentaba Fayol era una realidad laboral en donde las matemáticas no tenían mayor relevancia, por el contrario, las capacidades necesarias de los jefes, a medida que ascendían en la escala jerárquica apuntaban hacia sólidas prácticas administrativas, de ahí la necesidad de contar con el dominio de la ciencia administrativa (Fayol, 1977).

La administración industrial y general fue asimilada dentro del modelo burocrático en los países anglosajones y no fue traducida al inglés sino hasta 1929, mientras que Taylor fue traducido a varios idiomas de inmediato. En América Latina se han publicado ambas obras en un solo volumen desde la década de 1960, gozando de una amplia difusión y aplicación en las empresas y en la administración pública. En cuanto a Jaques, su libro Requisite organization fue traducido al español en Argentina en el año 2000.

Jaques encontró que una gran proporción de las empresas por él estudiadas, por múltiples razones, incluyendo la ignorancia, no contaba con el personal idóneo en los puestos de trabajo, por lo que las organizaciones experimentaban malestar y malos resultados. Los directivos se esforzaban en paliar esta condición adoptando modas administrativas introducidas por asesores que solamente funcionaban durante algún tiempo, para luego volver al mismo estado de cosas. Se lamentaba de que una democracia de libre empresa que no es capaz de proveer oportunidad para sus ciudadanos para utilizar sus capacidades potenciales plenas es una sociedad despilfarrada (Jaques, 2006). Proponía que solamente aplicando las herramientas científicas del alcance temporal y la capacidad de procesamiento mental, aunado a una correcta la aplicación del sistema burocrático, se tendrían resultados positivos y duraderos. Mientras que Fayol y Taylor provenían de una formación en el área de ingeniería, y por lo tanto enfatizaban (en particular Taylor) los aspectos relacionados con los procesos productivos, Jaques, 
con una formación médica y psicológica se enfocaba en el ser humano como eje de todo el engranaje de la organización.

Cuando inició la intervención en Glacier, Jaques conocía bien que es a partir de la ansiedad paranoica de quien recibe órdenes y de la ansiedad depresiva de quien da las órdenes (fuerzas dinámicas que llevan a los individuos hacia las organizaciones), que las instituciones son utilizadas inconscientemente por sus miembros como mecanismos de defensa contra esas ansiedades psicóticas, y ese fue el punto de partida de su investigación (Perret, 2009). De ahía la organización requerida pasaron muchos años de trabajo, el autor fue paulatinamente ampliando su enfoque hacia la estructura y los procesos; hacia la ingeniería, las finanzas y el marketing; hacia la organización en su totalidad, para al final presentar una teoría coherente y universal.

A diferencia de los otros dos autores, la organización requerida abarca a toda la jerarquía organizacional y a todo tipo de organizaciones, estudiando con detalle cada parte de la estructura, partiendo de los estratos I y II, que en las grandes empresas corresponden al trabajo operativo de línea realizado por los operarios y sus supervisores, tanto en el taller como en las oficinas (en las pymes comprende la actividad del propietario y sus empleados), hasta los estratos VI y VII, propios de las grandes multinacionales, en donde todas las funciones están diferenciadas, con unidades de negocios organizadas en portafolios de vicepresidentes y funciones de staff corporativo estratégicas. Jaques describe el incremento de complejidad que se va dando a medida que se escala por los diferentes estratos. La complejidad la define el autor en términos de las variables que deben ser contempladas al mismo tiempo en una situación dada, la claridad y precisión con que deben ser identificadas y su tasa de cambio. La complejidad se ve afectada por los conocimientos y la tecnología. La complejidad va desde sobrepasar obstáculos con ayuda del juicio práctico en el estrato I hasta construir sistemas complejos en el estrato VII. Los problemas vienen empacados en categorías o estados de complejidad problemática, siendo diferente en calidad cada categoría superior de la categoría inferior, y considerando que la complejidad de un problema no reside en la complejidad de la meta, sino en la complejidad del camino que conduce a la meta (Jaques, 2006).

Las investigaciones de Jaques merecen ser conocidas por los estudiantes de áreas administrativas y directivos de empresas, su obra puede ser continuada y proyectada hacia la tercera revolución industrial, en especial en el área de Recursos Humanos, en donde su aplicación puede traer grandes beneficios al elevar la productividad y dar la oportunidad a los seres humanos de ejercer sus talentos y vivir una vida laboral plena y satisfactoria.

\section{REFERENCIAS}

Fayol, H. (1977). Administración industrial y general. México: Herrero Hermanos.

Foster, T. (2016). Levels of work and population statistics. [Video]. Retrieved from https://www.youtube. $\mathrm{com} /$ watch? $\mathrm{v}=6 \mathrm{gx}-\mathrm{yl} 10 \mathrm{Eex}$ U\&index $=7 \&$ list=PL0 ADD4CCF8627BFFE

Foster, T. (2016). Potential progression chart. [Video]. Retrieved from https://www.youtube.com/ watch? $v=6 g x-y 10$ EexU\&index=7\&list=PL0ADD 4CCF8627BFFE

Fullerton, K. (1959). Calvinism and capitalism: an explanation of the W Thesis. In R. W. Green (ed.). Protestantism and Capitalism: The Weber Thesis and its critics. New York: Macmillan. 
George, C., y Álvarez, L. (2005). Historia del pensamiento administrativo. México: Pearson Education.

Glaser, B., \& Strauss, A. (1967). The discovery of grounded theory: Strategies for qualitative research. New Brunswick, USA: Aldine Transaction.

Herzberg, F. (1966). Work and the nature of man. New York: The World Publishing Company.

Jaques, E. (2000). A general theory of bureaucracy. Portsmouth, NH, USA: Heinemann Educational Books, Inc.

Jaques, E., \& Cason, K. (2013). Human capability. Falls Church, VA, USA: Cason Hall \& Co. Publishers.

Jaques, E. (2006). Requisite organization. Baltimore, MD, USA: Cason Hall \& Co. Publishers.

Jaques, E. (1951). The changing culture of a factory. Londres: Tavistock Institute.
Kobayashi, S. (1972). Administración creativa. México: Editorial Técnica.

Peci, A. (2009). Taylorism in the socialsm that really existed. Organization Connexions, 16(2), 289-301.

Perret, V. (2009). De l' organization comme un moyen de lutte contre l'anxiété a la "Requisite Orsanization". EMS.

ROII, R. O. (2017). Elliott Jaques: The man, the science and the legacy. Fleming Island, FL, USA: ROII.

Taylor, F. (1972). Los principios de la administración cientifica. México: Herrero Hermanos.

Vidal, M. (2013). Posfordism as a dysfunctional accumulation regime: A comparative analysis of the USA, the UK and Germany. Work, Employment and Society, 27(3), 451-471. 